\title{
BMJ Open Defining and measuring suspicion of sepsis: an analysis of routine data
}

\author{
Matthew Inada-Kim, ${ }^{1}$ Bethan Page, ${ }^{2}$ Imran Maqsood, ${ }^{3}$ Charles Vincent ${ }^{2}$
}

To cite: Inada-Kim M, Page B, Maqsood I, et al. Defining and measuring suspicion of sepsis: an analysis of routine data. BMJ Open 2017;7:e014885. doi:10.1136/ bmjopen-2016-014885

- Prepublication history and additional material are available. To view these files please visit the journal online (http://dx.doi. org/10.1136/bmjopen-2016014885).

Received 24 October 2016 Revised 13 January 2017 Accepted 4 May 2017

\section{CrossMark}

${ }^{1}$ Hampshire Hospitals NHS Foundation Trust, Royal Hampshire County Hospital, Winchester, UK

${ }^{2}$ Department of Experimental Psychology, University of Oxford, Oxford, UK

${ }^{3}$ Oxford Academic Health Science Network, Oxford Science Park, Magdalen Centre, Oxford, UK

Correspondence to Dr Matthew Inada-Kim; mattinadakim@hotmail.com

\begin{abstract}
Objectives To define the target population of patients who have suspicion of sepsis (SOS) and to provide a basis for assessing the burden of SOS, and the evaluation of sepsis guidelines and improvement programmes.

Design Retrospective analysis of routinely collected hospital administrative data.

Setting Secondary care, eight National Health Service (NHS) Acute Trusts.

Participants Hospital Episode Statistics data for 20132014 was used to identify all admissions with a primary diagnosis listed in the 'suspicion of sepsis' (SOS) coding set. The SOS coding set consists of all bacterial infective diagnoses.

Results We identified 47475 admissions with SOS, equivalent to a rate of 17 admissions per 1000 adults in a given year. The mortality for this group was $7.2 \%$ during their acute hospital admission. Urinary tract infection was the most common diagnosis and lobar pneumonia was associated with the most deaths. A short list of 10 diagnoses can account for $85 \%$ of the deaths.

Conclusions Patients with SOS can be identified in routine administrative data. It is these patients who should be screened for sepsis and are the target of programmes to improve the detection and treatment of sepsis. The effectiveness of such programmes can be evaluated by examining the outcomes of patients with SOS.
\end{abstract}

\section{INTRODUCTION}

Sepsis, defined as a "life-threatening organ dysfunction caused by a dysregulated host response to infection', 'is one of the leading causes of death. ${ }^{23}$ The incidence of sepsis is thought to be increasing, ${ }^{4-7}$ with estimates of up to 300 cases per 100000 population, ${ }^{8}$ and it has been recently cited as the most expensive reason for hospitalisation in the USA. ${ }^{9}$ Rapid, evidence-based, aggressive treatment and assiduous review are vital for preserving postrecovery function and ensuring survival. Sepsis is most common in the elderly and those with impaired immune systems and is associated with reduced quality of life and high rates of late mortality in those who survive. ${ }^{1011}$

Worldwide awareness of sepsis has been increasing due to high-profile media attention, coupled with reports from the surviving sepsis campaign and a multitude of national

\section{Strengths and limitations of this study}

- Our methodology for identifying suspicion of sepsis (SOS) uses routine administrative data, providing a means of assessing the SOS burden and comparing patient outcomes over time and across organisations.

- Monitoring the outcomes of patients with SOS provides a simple and effective means of measuring the impact of sepsis improvement programmes.

- The analysis allowed the most common and the most high-risk infections to be identified.

- The mortality figures are limited to in-hospital deaths: 30 -day mortality data were unavailable.

- The estimates for the SOS burden are based on Hospital Episode Statistics data which ultimately may not be as accurate as prospective data based on clinical and physiological measurements.

regulators and expert bodies. ${ }^{12-15}$ The early detection and treatment of sepsis has been highlighted as a major focus for improvement. For example, in the UK, the identification and early treatment of sepsis is the target of a major national campaign and also the focus for Commissioning for Quality and Innovation for 2015-2018, a financial incentive system. ${ }^{16}$ The lack of suitable metrics for sepsis has hampered evaluation and some have suggested that until this is resolved, no sepsis campaigns should be launched. ${ }^{17}$

\section{Measures of the incidence of sepsis}

There are three broad approaches to assessing the incidence of sepsis: through laboratory analytic tests such as blood cultures, through clinical judgement and through administrative databases (essentially the recording of clinical judgement). Many patients with sepsis do not have currently identifiable bacterial growth in blood tests (positive blood cultures), meaning that these investigations alone cannot assess the incidence of sepsis. ${ }^{18}$ Clinical studies of sepsis have been primarily focused on intensive care units (ICUs) and suggest that the incidence of severe sepsis in intensive care is approximately $30 \% .{ }^{20}{ }^{21}$ However, these studies, while 
informative in the intensive care environment, do not address the incidence of sepsis in other clinical settings within the hospital which is where most improvement programmes are now focused and where the majority of septic patients are managed.

The most practical means of assessing the incidence of sepsis in the wider hospital environment is through the analysis of administrative databases. However, there is great variability in estimates of sepsis incidence depending on the coding set used ${ }^{22} 23$ and changes in coding practices overtime make comparisons between years difficult. ${ }^{172224}$ In the UK, clinicians rarely document 'sepsis or septicaemia' in the admission documentation and tend to prioritise documenting the source of infection. ${ }^{12}{ }^{13}$ Official estimates for sepsis incidence, such as the Hospital Episode Statistics figures reported by National Health Service (NHS) England, are broadly based on septicaemia codes, ${ }^{5}$ and therefore underestimate the true incidence. Studies in the USA frequently report rising sepsis incidence and falling mortality: it is unclear whether the falling mortality is due to actual improvements in care or a diluting effect of increased coding of patients who are less sick than those who would previously have been included. ${ }^{25}$ In any case, the wider aim is not so much the treatment of sepsis as the prevention of sepsis through early intervention in patients who are at risk of sepsis.

\section{Suspicion of sepsis}

The concept of assessing and treating patients with suspected sepsis is central to the recently published National Institute for Health and Care Excellence (NICE) guidelines, with clinicians urged to think sepsis for all patients with signs of infection. ${ }^{26}{ }^{27}$ In practice, clinicians do not wait to diagnose sepsis but rapidly treat patients with an infection that is serious enough to warrant hospital admission; they endeavour to intervene to prevent full-blown sepsis developing and to interrupt the dangerous dysregulated and harmful immune response that may emerge. Some of these patients will of course already have a 'bad or serious infection' that is sufficient to warrant the term 'sepsis'. Most sepsis campaigns and improvement programmes do not actually target the treatment of fully developed sepsis but instead are aimed at the rapid detection and treatment of patients who have suspected sepsis when admitted to hospital from the community. This means that we must, first and foremost, identify those patients who either have early sepsis or who have an infection which might develop into sepsis if not properly treated.

The group, 'suspicion of sepsis', consists primarily of patients who have a bacterial infection serious enough to require hospital admission. ${ }^{28}{ }^{29}$ Defining this group is essential to the implementation of NICE guidelines and to all the recommendations for research proposed in the guidelines. ${ }^{26}$ Although there are non-bacterial causes of sepsis (eg, viruses, protozoa), these are (generally) far less common and amenable to treatment. Given that the majority of septic patients have community-acquired infections, ${ }^{30}$ our focus, and that of most sepsis improvement programmes, will be on patients with infections at the time of admission. To evaluate programmes aimed at early detection and intervention, we must therefore define a 'suspicion of sepsis' group and monitor the progress of its members. A successful intervention would see a reduction in later sepsis (however measured), but even in the absence of reliable sepsis definition, we could monitor mortality, length of stay (LOS) and other indices of outcome in a suspicion of sepsis (SOS) group.

\section{Identifying patients with 'suspicion of sepsis'}

The primary aim of this study is to estimate the number of patients with SOS on admission to hospital and their outcomes (mortality, LOS, readmission rate). We focus on patients who either have bacterial infections which may lead to sepsis or which have already led to early sepsis. Analysis of a full list of the International Statistical Classification of Diseases and Related Health Problems Tenth Revision (ICD-10) diagnostic infection codes that can cause sepsis will provide clinical insight into which infections are the most frequent, which are the most dangerous and which might be prioritised for future improvement strategies.

\section{METHOD}

A list of ICD-10 (2015) codes was developed for infective bacterial pathogens that can cause sepsis (see online supplementary appendix A). The coding set entitled 'Suspicion of Sepsis' was established on the basis of clinical consensus: a consultant in acute medicine (MIK) reviewed the full list of ICD-10 codes and identified all codes known to be infective bacterial pathogens, requiring treatment with antibiotics. Specialist consultants for each organ system were then asked to verify the list of codes relevant to their specialty and the codes were subsequently adjusted based on their feedback. The list also includes specific sepsis codes (ICD-10 codes A40 and A41 and their derivatives) which are commonly used in the UK for instances of sepsis where the source of infection is unknown. ${ }^{12} 13$

Hospital Episode Statistics (HES) data were obtained for all acute trusts in the Oxford Academic Health Science Network (AHSN) region $(n=8)$ for the financial year 2013-2014: the region covers a population of 3.3 million. The data originated from the Health and Social Care Information Centre. A data warehouse was created in Microsoft SQL Server for running queries and completing data analysis.

We analysed the admission episode to determine if any of the ICD-10 'Suspicion of Sepsis' codes appeared as the primary diagnosis. Coders use information from patient notes to retrospectively determine the primary reason for admission. Patients under the age of 18 were excluded. For each identified admission, the following information was determined: age at admission, sex, number of hospital deaths and associated mortality, LOS and readmission 


$$
6000
$$

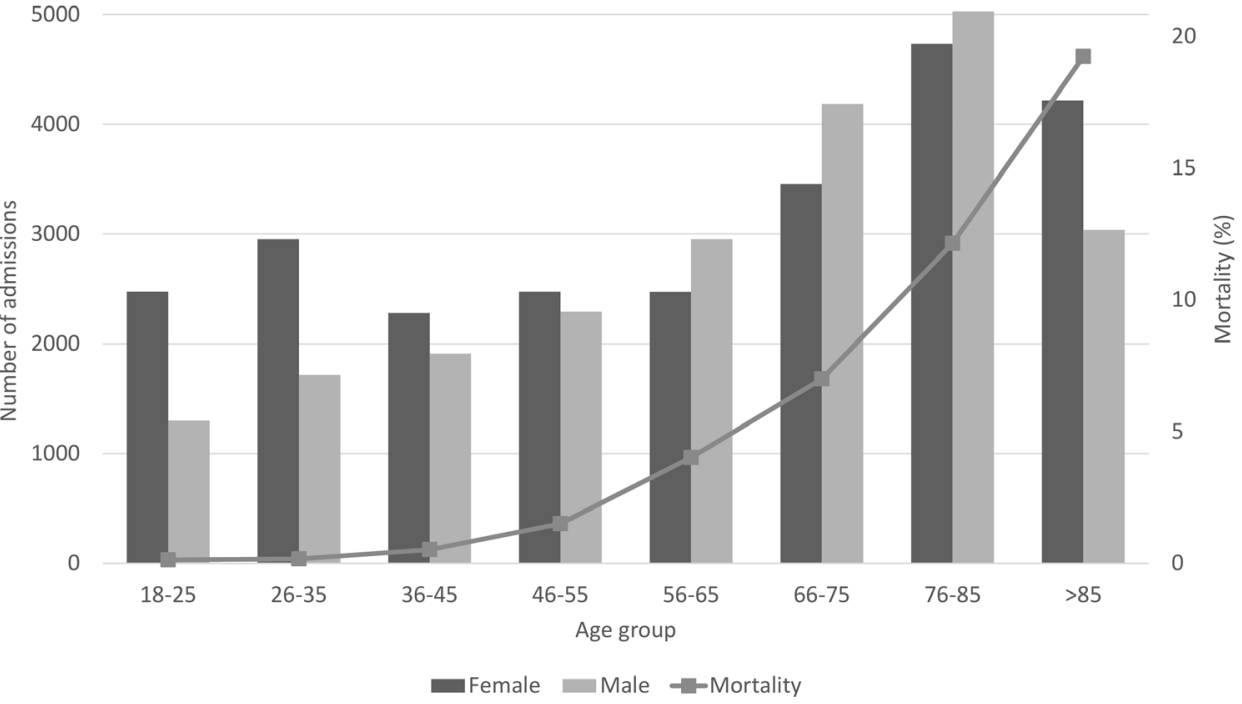

Figure 1. Suspicion of sepsis admissions and mortality by age and gender.

rate. ${ }^{*}$ Population data provided by the Clinical Commissioning Groups which fall within the Oxford AHSN region were used to estimate a population incident rate. A short practical guide for others wanting to replicate our methodology is available in supplementary appendix A.

\section{Patient involvement}

Patient experiences guided the principles of this study: a consistent theme in patient stories and local reviews of sepsis cases is that early symptoms were not recognised. Patients were not involved in the study design or the technical analysis of administrative databases. The concepts of the paper have been presented to both patients and carers in the context of measuring the impact of sepsis improvement programmes.

\section{RESULTS}

\section{Incidence and demographics}

In 2013-2014, 47475 admissions were identified in the Oxford AHSN region using the 'suspicion of sepsis' coding set, yielding a population estimate of 17 SOS hospital admissions per 1000 adults in a given year. The overall in-hospital mortality rate for this group was $7.2 \%$, which represents 3440 deaths. The mean LOS was 9.2 days and $6.7 \%$ of patients with SOS were readmitted within 30 days.

The number of SOS admissions by age and gender and the mortality rate by age are shown in figure 1 . There was no missing data. The patient was female in 52.8\% of admissions. The number of admissions increased

\footnotetext{
* A patient was classified as a readmission if they were admitted as an emergency readmission between 1 and 30 days after their previous discharge. The main specialty of the two spells needed to match in order to be classified as a readmission.
}

gradually with age, before decreasing for over $85 \mathrm{~s}$. Women between 18 and 35 (childbearing age) were almost twice as likely as men of the same age to have suspected sepsis and women over 85 also had a higher incidence than men, likely as a result of the higher female population in comparison with men over 85 years. For all other age groups, the number of SOS admissions in men and women were similar. Hospital mortality increased with age. The mortality rate was less than $1 \%$ for all age groups up to aged 45. From 46 upwards, the mortality increased exponentially: for patients over 85 , the mortality was $19.2 \%$.

\section{Most common diagnoses}

The following four ICD-10 chapters combined account for almost $85 \%$ of SOS admissions: 'Diseases of the Respiratory System' (39.8\%), 'Diseases of the Digestive System' (11.1\%), 'Diseases of Genitourinary System' (21.0\%) and 'Diseases of Skin and Subcutaneous Tissue' (12.5\%). Two thousand five hundred and seventy-seven $(5.4 \%)$ of the SOS admissions had a sepsis code (a code commencing A40 or A41) as their primary diagnosis.

Table 1 lists the 25 most common diagnoses, alongside their respective mortality rates, number of deaths, LOS and readmission rates. A complete list of all the suspicions of sepsis diagnoses with the number of admissions, number of deaths, mortality, LOS and readmission rate is presented in online supplementary appendix B. The majority of the diagnoses in table 1 are infections of the respiratory system. The most common diagnosis was urinary tract infection. A41.9 sepsis, the most common of the sepsis codes, was the seventh most common SOS diagnosis.

The secondary aim of this study was to develop a short set of codes which could be easily tracked. The 25 
Table 1 Top 25 most common 'Suspicion of Sepsis' diagnoses in Oxford Academic Health Science Network region (20132014)

\begin{tabular}{|c|c|c|c|c|c|}
\hline Diagnosis & Admissions (n) & Deaths (n) & $\begin{array}{l}\text { Mortality } \\
(\%)\end{array}$ & LOS & $\begin{array}{l}\text { Readmission } \\
(\%)\end{array}$ \\
\hline 1. N39.0-Urinary tract infection, site not specified & 7088 & 285 & 4.0 & 9.0 & 6.4 \\
\hline 2. J18.1-Lobar pneumonia, unspecified & 5265 & 990 & 18.8 & 9.8 & 6.3 \\
\hline 3. J22.X-Unspecified acute lower respiratory infection & 3808 & 167 & 4.4 & 6.5 & 7.4 \\
\hline 4. J18.9-Pneumonia, unspecified & 3126 & 483 & 15.5 & 9.6 & 7.1 \\
\hline 5. L03.1-Cellulitis of other parts of limb & 2983 & 62 & 2.1 & 6.9 & 7.5 \\
\hline $\begin{array}{l}\text { 6. J44.0-Chronic obstructive pulmonary disease with } \\
\text { acute lower respiratory infection }\end{array}$ & 2854 & 184 & 6.4 & 7.2 & 11.3 \\
\hline 7. A41.9-Sepsis, unspecified & 1882 & 280 & 14.9 & 9.2 & 10.7 \\
\hline $\begin{array}{l}\text { 8. T81.4-Infection following a procedure, not } \\
\text { elsewhere classified }\end{array}$ & 1067 & 3 & 0.3 & 5.9 & 7.7 \\
\hline 9. J03.9-Acute tonsillitis, unspecified & 1055 & 0 & 0.0 & 1.2 & 5.7 \\
\hline 10. K35.8-Acute appendicitis, other and unspecified & 993 & 0 & 0.0 & 2.9 & 6.4 \\
\hline $\begin{array}{l}\text { 11. N12.X-Tubulo-interstitial nephritis, not specified } \\
\text { as acute or chronic }\end{array}$ & 809 & 1 & 0.1 & 3.5 & 3.5 \\
\hline 12. K61.0-Anal abscess & 781 & 0 & 0.0 & 1.2 & 5.6 \\
\hline 13. J69.0-Pneumonitis due to food and vomit & 776 & 260 & 33.5 & 14.1 & 5.0 \\
\hline $\begin{array}{l}\text { 14. L02.4-Cutaneous abscess, furuncle and } \\
\text { carbuncle of limb }\end{array}$ & 710 & 0 & 0.0 & 2.5 & 5.6 \\
\hline 15. L05.0-Pilonidal cyst with abscess & 533 & 0 & 0.0 & 0.6 & 4.9 \\
\hline $\begin{array}{l}\text { 16. L02.2-Cutaneous abscess, furuncle and } \\
\text { carbuncle of trunk }\end{array}$ & 527 & 1 & 0.2 & 2.5 & 8.2 \\
\hline $\begin{array}{l}\text { 17. O23.4-Unspecified infection of urinary tract in } \\
\text { pregnancy }\end{array}$ & 518 & 0 & 0.0 & 1.3 & 1.0 \\
\hline $\begin{array}{l}\text { 18. K80.0-Calculus of gallbladder with acute } \\
\text { cholecystitis }\end{array}$ & 517 & 6 & 1.2 & 4.7 & 8.5 \\
\hline 19. K37.X-Unspecified appendicitis & 505 & 1 & 0.2 & 2.5 & 3.8 \\
\hline 20. J18.0-Bronchopneumonia, unspecified & 432 & 242 & 56.0 & 10.5 & 5.8 \\
\hline 21. J36.X-Peritonsillar abscess & 429 & 0 & 0.0 & 1.3 & 2.8 \\
\hline $\begin{array}{l}\text { 22. N45.9-Orchitis, epididymitis and epididymo- } \\
\text { orchitis without abscess }\end{array}$ & 413 & 0 & 0.0 & 1.8 & 6.1 \\
\hline $\begin{array}{l}\text { 23. A41.5-Sepsis due to other Gram-negative } \\
\text { organisms }\end{array}$ & 360 & 40 & 11.1 & 11.5 & 10.8 \\
\hline $\begin{array}{l}\text { 24. T84.5-Infection and inflammatory reaction due to } \\
\text { internal joint prosthesis }\end{array}$ & 345 & 4 & 1.2 & 19.1 & 5.5 \\
\hline \multirow[t]{2}{*}{ 25. K81.9-Cholecystitis, unspecified } & 339 & 5 & 1.5 & 4.0 & 8.8 \\
\hline & $n=38115$ & $n=3014$ & $m=6.9$ & $m=6.0$ & $\mathrm{~m}=6.5$ \\
\hline
\end{tabular}

LOS, length of stay.

diagnoses in table 1 capture $80.3 \%$ of the total number of SOS admissions and $87.6 \%$ of the deaths. These 25 diagnoses include a number of diagnoses which are common but rarely lead to poor outcomes.

\section{Diagnoses associated with the most deaths}

The most important patients to identify and track in improvement programmes are those who have the poorest outcomes. Table 2 lists the 10 diagnoses associated with the most deaths. Lobar pneumonia was associated with the most deaths, followed by pneumonia unspecified and then urinary tract infection. A41.9 sepsis was fourth on the list. Respiratory infections featured frequently in table 1 : indeed diagnoses from the ICD-10 chapter 'Diseases of the Respiratory System' accounted for $69.8 \%$ of the total number of SOS deaths.

Together, the 10 diagnoses listed in table 2 account for $87.3 \%$ of the total number of SOS deaths and $59.7 \%$ of the total number of SOS admissions. The mortality for this group of 10 diagnoses is $10.6 \%$, the mean LOS is 9.6 days and the readmission rate is $7.3 \%$ : these are much 
Table 2 Top 10 'Suspicion of Sepsis' diagnoses associated with death in Oxford Academic Health Science Network region (2013-2014)

\begin{tabular}{|c|c|c|c|c|c|}
\hline Diagnosis & Deaths (n) & Admissions (n) & $\begin{array}{l}\text { Mortality } \\
(\%)\end{array}$ & LOS & $\begin{array}{l}\text { Readmission } \\
(\%)\end{array}$ \\
\hline 1. J18.1-Lobar pneumonia, unspecified & 990 & 5265 & 18.8 & 9.8 & 6.3 \\
\hline 3. N39.0-Urinary tract infection, site not specified & 285 & 7088 & 4.0 & 9.0 & 6.4 \\
\hline 4. A41.9-Sepsis, unspecified & 280 & 1882 & 14.9 & 9.2 & 10.7 \\
\hline $\begin{array}{l}\text { 7. J44.0-Chronic obstructive pulmonary disease with } \\
\text { acute lower respiratory infection }\end{array}$ & 184 & 2854 & 6.4 & 7.2 & 11.3 \\
\hline 8. J22.X-Unspecified acute lower respiratory infection & 167 & 3808 & 4.4 & 6.5 & 7.4 \\
\hline 9. L03.1-Cellulitis of other parts of limb & 62 & 2983 & 2.1 & 6.9 & 7.5 \\
\hline
\end{tabular}

LOS, length of stay.

poorer outcomes than for the 25 more common diagnoses where the mortality is $7.9 \%$, the mean LOS is 6.0 days and the mean readmission rate is $6.5 \%$. figure 2 shows a visual representation of these 10 high-risk diagnoses, their frequency, their outcomes (mortality and LOS) and the type of infection. Early intervention and treatment improvement programmes should aim to improve the outcomes for patients admitted to hospital with these 10 diagnoses.

\section{DISCUSSION}

Our methodology identifies those patients who should be routinely screened for sepsis on admission to hospital. Based on the identification of 47475 SOS admissions in Oxford AHSN region in the financial year 2013-2014, we estimate 17 SOS hospital admissions in a population of 1000 adults in a given year. Analysis of the individual SOS diagnoses allows a short pragmatic set of codes to be developed. For instance, the top 10 diagnoses associated

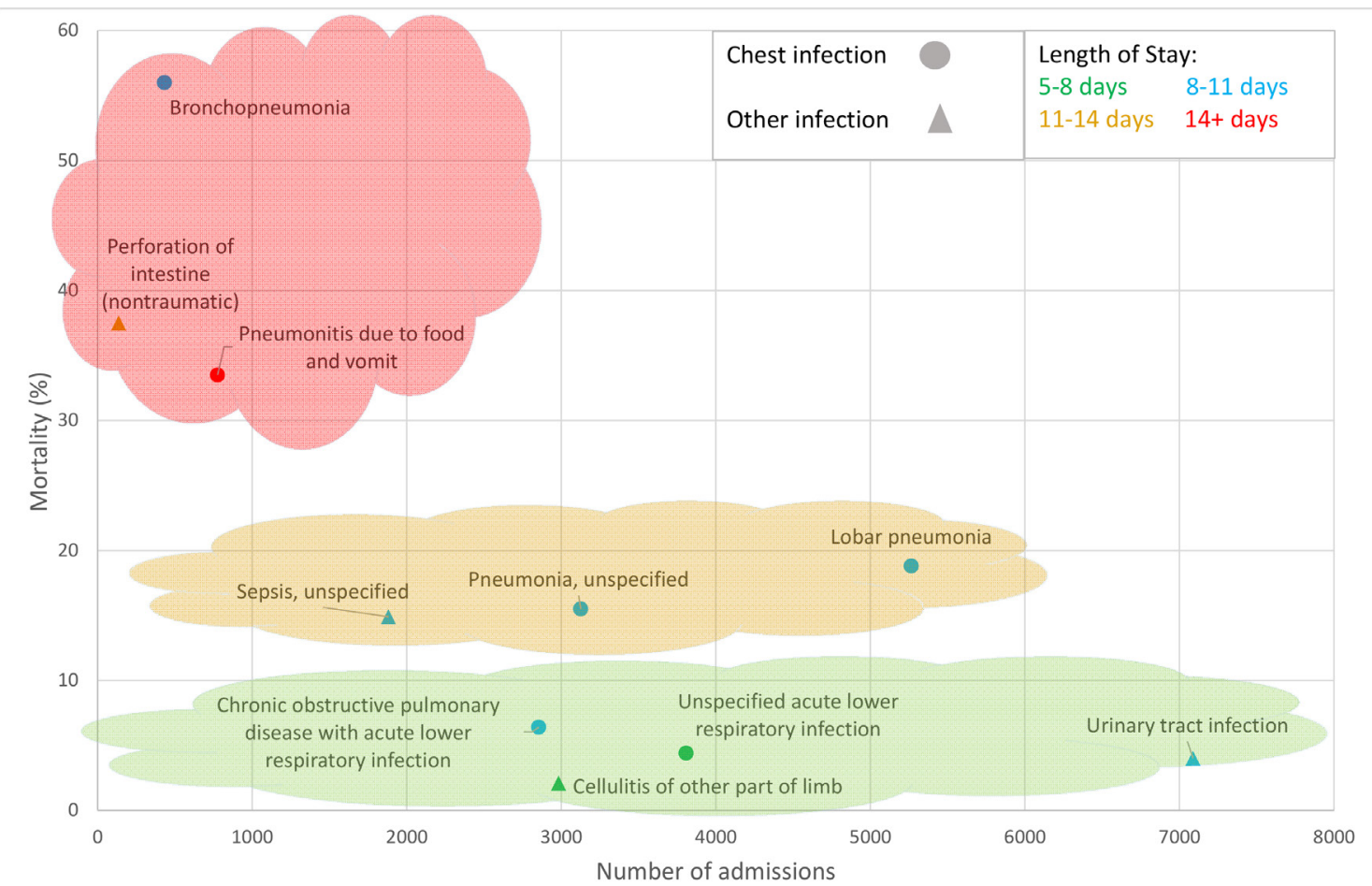

Figure 2. Top 10 'Suspicion of Sepsis' diagnoses associated with death. 
with the highest numbers of hospital deaths can account for $85 \%$ of the total number of SOS deaths: monitoring the outcomes of patients admitted to hospital with one of these 10 crucial diagnoses (eg, mortality, LOS, readmission rate) would be a pragmatic and effective means of assessing sepsis campaigns and improvement efforts.

Previous studies have found that the number of recorded septic episodes has artificially increased as a result of increased coding rather than solely more cases of sepsis, particularly in the USA. ${ }^{24}$ By tracking infective diagnoses, our methodology avoids this bias and hence would be a more effective measure of improvement over longer time periods. Clinical specialties are also more easily able to identify and track patients who are particularly relevant to them. Different countries could also use the methodology from this study to identify the most common and most fatal SOS diagnoses in their countries. The full SOS coding set could also be used to compare figures internationally for the population with SOS.

\section{Future research and evaluation}

We have been able to estimate the size of the SOS population on admission to hospital in our region; this should now be done nationally. Ideally, we would also want to measure the percentage of SOS cases who develop definite sepsis, which would mean we would have a means of evaluating campaigns and programmes which aim to improve the detection and early treatment of potential sepsis. However, major improvements are needed in the quality of coding of sepsis if we are to use ICD codes to identify definite cases of sepsis. Even if we used case note review, evidence shows that diagnosing sepsis is subjective and variable. ${ }^{31}$ The exact size of the sepsis 'bubble' within the SOS set is likely to remain unknown in the absence of a definitive diagnostic test. However, linking the SOS database with electronic clinical observation data (eg, physiology and pathology blood results) may allow specific definitions of sepsis to be applied. A potential surrogate measure may be the proportion of patients with SOS admitted to intensive care.

Future studies can use the SOS population to study predictors of poor outcomes, for example, by linking the SOS database with electronic clinical observation data. SOS could also be used to assess the usefulness of potential biomarkers and to assess the impact of improvement campaigns, from small-scale quality improvement work to national strategies such as the new NICE guidelines. Future studies should also estimate the costs and associated bed days for SOS.

\section{Limitations}

Our clinical assumption in reporting any-cause mortality for SOS diagnoses is that the presence of infection has a major impact on outcomes, above and beyond other factors such as comorbidities, but this ought to be tested. Furthermore, our mortality figures are based on HES data and are therefore limited to in-hospital deaths: future studies should also endeavour to assess 30-day mortality. Our estimates for the size of the SOS population are based on ICD codes which ultimately may not be as accurate as prospective data based on clinical and physiological measurements. However, our approach can be easily and cheaply used by any organisation to identify and monitor this critical group of patients. Our methodology measures SOS on admission; it does not address patients who develop an infection during their hospital stay. It is also worth noting that our estimates are based on one area of the country. Variability between regions is likely to be affected by such factors as social economic conditions, the quality of the hospitals and the average age of the population. Our figures for the population incidence with SOS do suggest however that the national burden is substantial.

\section{Conclusions}

We propose that patients admitted to hospital for infection ('Suspicion of Sepsis') are a critical target population both for screening for sepsis and for monitoring the impact of sepsis improvement efforts. This group can be easily identified from routine administrative data. Analysis of local UK data yields an estimate of 17 hospital admissions with SOS per 1000 adults in a given year and revealed that a short list of 10 diagnoses can capture $85 \%$ of SOS deaths. Monitoring the outcomes of patients with SOS is a simple and effective measurement strategy for evaluating programmes aiming to improve the detection and treatment of sepsis.

Acknowledgements We thank the Oxford AHSN for acquiring the HES data used in this study.

Contributors MIK conceived the study. IM carried out the analysis and BP prepared all the tables and figures. BP and CV prepared the first draft of the paper. All authors provided critical feedback and contributions to the final paper.

Funding The work was supported by grants from Oxford Academic Health Science Network. The funder enabled access to the data. They had no role in study design, data analysis, data interpretation or writing of the report. The corresponding author had full access to all the data in the study and had final responsibility for the decision to submit for publication.

Competing interests MIK reports grants for roles as clinical lead for sepsis across Oxford Academic Health Science Network and clinical lead of the Patient Safety Collaborative, Wessex. BP and IM declare no competing interests. CV declares grants from Oxford Academic Health Science Network, during the conduct of the study and occasional consultancy work on patient safety unrelated to this project.

Patient consent Detail has been removed from this case description/these case descriptions to ensure anonymity. The editors and reviewers have seen the detailed information available and are satisfied that the information backs up the case the authors are making.

Ethics approval Not required as the data were routinely collected hospital administrative data and not in a patient identifiable format.

Provenance and peer review Not commissioned; externally peer reviewed.

Data sharing statement A full list of the suspicion of sepsis ICD-10 codes is given in Appendix A. Additional information on methodology can be obtained from the corresponding author on request. No additional data is available. The Hospital Episode Statistics (HES) data in this study was used with the permission of The Health \& Social Care Information Centre. All rights reserved.

Open Access This is an Open Access article distributed in accordance with the Creative Commons Attribution Non Commercial (CC BY-NC 4.0) license, which permits others to distribute, remix, adapt, build upon this work non-commercially, and license their derivative works on different terms, provided the original work is 
properly cited and the use is non-commercial. See: http://creativecommons.org/ licenses/by-nc/4.0/

(C) Article author(s) (or their employer(s) unless otherwise stated in the text of the article) 2017. All rights reserved. No commercial use is permitted unless otherwise expressly granted.

\section{REFERENCES}

1. Singer M, Deutschman CS, Seymour CW, et al. The third international consensus definitions for sepsis and septic shock (sepsis-3). JAMA 2016;315:801-10.

2. Xu J, Kenneth D, Kochanek MA, et al. Deaths: final data for 2007 . National Vital Statistics Reports 2010;58.

3. Elixhauser A, Friedman B, Stranges E. Septicemia in US hospitals, 2009: Statistical Brief \#122. Healthcare Cost and Utilization Project (HCUP) Statistical Briefs [Internet] 2011.

4. Harrison DA, Welch CA, Eddleston JM. The epidemiology of severe sepsis in England, Wales and Northern Ireland, 1996 to 2004: secondary analysis of a high quality clinical database, the ICNARC case mix programme database. Crit Care 2006;10:R42.

5. Health and Social Care Information Centre. Hospital episode statistics. 2015 http://www.hscic.gov.uk/media/19070/Sepsisannual-data-inpatient-measures/xls/Sepsis_annual_data_inpatient measures_(1).xlsx (accessed 3 Mar 2016).

6. Dombrovskiy VY, Martin AA, Sunderram J, et al. Rapid increase in hospitalization and mortality rates for severe sepsis in the United States: a trend analysis from 1993 to 2003. Crit Care Med 2007;35:1244-50.

7. Kumar G, Kumar N, Taneja A, et al. Nationwide trends of severe sepsis in the 21st century (2000-2007). Chest 2011;140:1223-31.

8. Angus DC, Linde-Zwirble WT, Lidicker J, et al. Epidemiology of severe sepsis in the United States: analysis of incidence, outcome, and associated costs of care. Crit Care Med 2001;29:1303-10.

9. Torio CM, Andrews RM. National inpatient hospital costs: the most expensive conditions by payer. 2011;160. Statistical brief no. https:// www.hcup-us.ahrq.gov/reports/statbriefs/sb160.jsp

10. Winters BD, Eberlein M, Leung J, et al. Long-term mortality and quality of life in sepsis: a systematic review. Crit Care Med 2010;38:1276-83.

11. Prescott HC, Osterholzer JJ, Langa KM, et al. Late mortality after sepsis: propensity matched cohort study. BMJ 2016;353:i2375.

12. NCEPOD. Just say sepsis! A review of the process of care received by patients with sepsis. 2015 http://www.ncepod.org.uk/ 2015report2/downloads/JustSaySepsis_FullReport.pdf (accessed 1 Feb 2016).

13. England NHS. Improving outcomes for patients with sepsis. 2015 https://www.england.nhs.uk/wp-content/uploads/2015/08/SepsisAction-Plan-23.12.15-v1.pdf
14. Parliamentary and Health Service Ombudsman. Time to act: severe sepsis: rapid diagnosis and treatment saves lives. http://www. ombudsman.org.uk/_data/assets/pdf_file/0004/22666/FINAL_ Sepsis Report web.pdf2013 (accessed 20 May 2016).

15. Dellinger RP, Levy MM, Rhodes A, et al. Surviving Sepsis Campaign: international guidelines for management of severe sepsis and septic shock, 2012. Intensive Care Med 2013;39:165-228.

16. England NHS. Commissioning for Quality and Innovation (CQUIN) Guidance for 2015/16. https://www.england.nhs.uk/wp-content/ uploads/2015/03/9-cquin-guid-2015-16.pdf (accessed 17 Feb 2016).

17. Rhee C, Gohil S, Klompas M. Regulatory mandates for sepsis care-reasons for caution. N Engl J Med 2014;370:1673-6.

18. Bernard GR, Vincent JL, Laterre PF, et al. Efficacy and safety of recombinant human activated protein $\mathrm{C}$ for severe sepsis. $N$ Engl $J$ Med 2001;344:699-709.

19. Phua J, Ngerng W, See K, et al. Characteristics and outcomes of culture-negative versus culture-positive severe Sepsis. Crit Care 2013;17:R202

20. Vincent JL, Sakr Y, Sprung CL, et al. Sepsis in European intensive care units: results of the SOAP study. Crit Care Med 2006;34:344-53.

21. Alberti $\mathrm{C}$, Brun-Buisson $\mathrm{C}$, Burchardi $\mathrm{H}$, et al. Epidemiology of sepsis and infection in ICU patients from an international multicentre cohort study. Intensive Care Med 2002;28:108-21.

22. Jolley RJ, Sawka KJ, Yergens DW, et al. Validity of administrative data in recording sepsis: a systematic review. Crit Care 2015;19:139.

23. Wilhelms SB, Huss FR, Granath G, et al. Assessment of incidence of severe sepsis in Sweden using different ways of abstracting International classification of diseases codes: difficulties with methods and interpretation of results. Crit Care Med 2010;38:1442-9.

24. Rhee C, Murphy MV, Li L, et al. Comparison of trends in sepsis incidence and coding using administrative claims versus objective clinical data. Clin Infect Dis 2015;60:88-95

25. Cohen J, Vincent JL, Adhikari NK, et al. Sepsis: a roadmap for future research. Lancet Infect Dis 2015;15:581-614.

26. National Institute for Health and Care Excellence (NICE). Sepsis: recognition, diagnosis and early management 2016 https://www.nice. org.uk/guidance/ng51 (accessed 15 Sept 2016).

27. Freitag A, Constanti M, O'Flynn N, et al. Suspected sepsis: summary of NICE guidance. BMJ 2016;354:i4030.

28. Danai P, Martin GS. Epidemiology of sepsis: recent advances. Curr Infect Dis Rep 2005;7:329-34.

29. Martin GS. Sepsis, severe sepsis and septic shock: changes in incidence, pathogens and outcomes. Expert Rev Anti Infect Ther 2012;10:701-6.

30. Esteban A, Frutos-Vivar F, Ferguson ND, et al. Sepsis incidence and outcome: contrasting the intensive care unit with the hospital ward. Crit Care Med 2007;35:1284-9.

31. Rhee C, Kadri SS, Danner RL, et al. Diagnosing sepsis is subjective and highly variable: a survey of intensivists using case vignettes. Crit Care 2016;20:89. 